\title{
Multi-mission observations of the old nova GK Per during the 2015 outburst
}

\author{
Polina Zemko* \\ Department of Physics and Astronomy, Universitá di Padova, vicolo dell'Osservatorio 3, \\ I-35122 Padova, Italy \\ E-mail: polina.zemkoestudenti.unipd.it

\section{Marina Orio} \\ INAF - Osservatorio di Padova, vicolo dell'Osservatorio 5, I-35122 Padova, Italy and \\ Department of Astronomy, University of Wisconsin, 475 N. Charter Str., Madison, WI 53704, \\ USA \\ E-mail: marina.oriodoapd.inaf.it
}

\section{Gerardo Juan Manuel Luna}

Instituto de Astronomía y Física del Espacio, (IAFE, CONICET-UBA), CC 67 - Suc. 28

(C1428ZAA) CABA - Argentina

E-mail: gjmluna@iafe.uba.ar

\section{Koji Mukai}

CRESST and X-ray Astrophysics Laboratory, NASA Goddard Space Flight Center, Greenbelt, MD 20771, USA and Department of Physics, University of Maryland, Baltimore County, 1000 Hilltop Circle, Baltimore, MD 21250, USA

E-mail: koji.mukai@umbc.edu

GK Per, a classical nova of 1901, is thought to undergo variable mass accretion onto a magnetized white dwarf (WD) in an intermediate polar system (IP). We organized a multi-mission observational campaign in the X-ray and ultraviolet (UV) energy ranges during its dwarf nova (DN) outburst in 2015 March-April. Comparing data from quiescence and near outburst, we have found that the maximum plasma temperature decreased from 26 to $16 \mathrm{keV}$. This is consistent with the previously proposed scenario of increase in mass accretion rate while the inner radius of the magnetically disrupted accretion disk shrinks, thereby lowering the shock temperature. A NuS$T A R$ observation also revealed a high-amplitude WD spin modulation of the very hard X-rays, suggesting an obscuration of the lower accretion pole and an extended shock region on the WD surface. In the Chandra observation with the High Energy Transmission Gratings (HETG), we detected prominent emission lines, where the ratios of $\mathrm{H}$-like to He-like transition for each element indicate a much lower temperature than the underlying continuum. We suggest that the X-ray spectrum in the $0.8-2 \mathrm{keV}$ range results from emission from different regions of collisionally ionized plasma with a possible contribution from photoionization processes.

The Golden Age of Cataclysmic Variables and Related Objects - III

7-12 September 2015

Palermo, Italy

${ }^{*}$ Speaker. 


\section{Introduction}

GK Per underwent a nova explosion on 1901 February 21 [1] and after a long period of irregular fluctuations, in 1948, it started to behave like a DN, with a small amplitude (1 - 3 mag.) outbursts lasting for up to two months, repeated every $\simeq 26$ months [2]. The most widely accepted explanation of these outbursts is a repeating thermal instability in the inner part of the accretion disk (inside-out outbursts; see [3], [4] for application of the disk instability to GK Per). GK Per hosts a magnetic WD (first proposed by [5] and [2]), and, hence, the accretion disk is truncated by the magnetosphere of the WD that surprisingly does not prevent the instability. Watson et al. first discovered the X-ray modulation with the period of $351 \mathrm{~s}$, related to the WD spin [6].

The orbital period is quite long - $1.997 \mathrm{~d}[7]$ and the distance to the object is well defined $470 \mathrm{pc}$ [8]. The secondary is a $\mathrm{K} 2$ type subgiant with the mass of $0.25 \mathrm{M}_{\odot}$ ([9], [6]) and the mass of the primary is $\leq 0.72 \mathrm{M}_{\odot}[6]$.

On 2015 March 6.84 Dubovsky (VSNET-ALERT 18388) and Schmeer (VSNET-ALERT 18389) discovered that GK Per has started a new DN outburst and was at a magnitude 12.8. We proposed a multimission observation campaign in order to follow the evolution of the object during the outburst and to obtain X-ray spectra in a broad energy range, revealing the physical processes that take place in this binary system.

\section{Observation and data analysis}

We started the observations of the 2015 DN outburst of GK Per as soon as it became visible for Swift - on March 122015 and observed it almost until optical maximum. We obtained two exposures per day with $S$ wift for two weeks and one exposure per day for another two weeks. Coordinated NUSTAR and Chandra HETG observations were performed on April 4 2015, close to the optical maximum. The Swift X-Ray Telescope (XRT) and Ultraviolet Optical Telescope (UVOT) data were processed with the ftools package. We used the processed $S$ wift Burst Allert Telescope (BAT) data from the $S$ wift BAT transient monitor page [10]. We reduced the Chandra data with CIAO v.4.7 and the NUSTAR data with the standard nuproducts pipeline. All the light curves were extracted after the barycentric correction. The X-ray spectra were analysed and fitted using XSPEC v. 12.8.2. The list of the observations with the exposure times and count rates is presented in Table 1 .

\section{Results}

Figure 1 shows a comparison between the development of the outburst in the optical, UV and X-rays. The top panel is the optical light curve, which was obtained from The American Association of Variable Star Observers (AAVSO) ${ }^{1}$. The beginning of the outburst in the optical band was taken as a reference and is marked with the red dashed vertical line in all the panels. The maximum of the outburst in the optical band is also marked with the blue line. The second panel shows the $S$ wift UVOT data in different filters. All the UVOT light curves showed a gradual rise until almost a week before maximum. The images in the lower energy filter $-\mathrm{U}-$ and the last

\footnotetext{
${ }^{1}$ https://www.aavso.org/
} 
Table 1: Observational log

\begin{tabular}{l|ccc}
\hline \hline Instrument & Date $^{a}$ & Exp.(s) & Count rate $^{b}$ \\
\hline Swift XRT & $57093.15-57121.06$ & $373.6-2401.5$ & $0.67-2.14$ \\
Chandra MEG & 57116.83 & 69008 & $0.0751 \pm 0.0010$ \\
Chandra HEG & 57116.83 & 69008 & $0.1214 \pm 0.0013$ \\
NuSTAR FPMA & 57116.12 & 42340 & $3.665 \pm 0.009$ \\
NuSTAR FPMB & 57116.12 & 42340 & $3.626 \pm 0.009$ \\
\hline
\end{tabular}

Notes. ${ }^{a}$ Modified Julian Date. ${ }^{b}$ The count rates were measured in the following energy ranges: Chandra Medium Energy Grating (MEG) - 0.4-5.0 keV, Chandra High Energy Gratings (HEG) - 0.8-10.0 keV, NuSTAR Focal Plane Modules A and B (FPMA and FPMB) - 3-79 keV.

observations in the UVW1 and UVW2 filters were saturated and provide only lower limits for the magnitudes. The third panel is the Swift XRT light curve averaged within a snapshot in the whole energy range: from 0.3 to $10 \mathrm{keV}$. The count rate varied from 0.7 to $2.8 \mathrm{cnts} \mathrm{s}^{-1}$ but did not show any significant increasing or decreasing trend.

We missed the initial steep rise, observed only with $S$ wift BAT, because GK Per was too close to the sun, and observed only a plateau in the X-ray flux. In fig. 1, the bottom panels show the soft (0.3-2.0 keV) and hard (2.0-10 keV) XRT light curves. The hard count rate is more scattered in comparison with the soft one and decreased as the outburst developed. The soft count rate, in turn, showed a prominent rise, which resulted in a gradual decrease of the hardness ratio (panel 5) with minimum around MJD 57113, 25 days after the beginning of the outburst. The light curve measured with the $S$ wift BAT is more stable and only showed a moderate decrease after maximum around day 10 after the outburst. The flux increase started 2 days earlier in the $S$ wift BAT energy range than in optical.

\subsection{Timing analysis}

For our timing analysis we extracted the $S$ wift XRT, NuSTAR and Chandra light curves and binned them every $10 \mathrm{~s}$. In order to investigate a possible energy dependence of the X-ray variability we split the Swift XRT light curve in two ranges: $0.3-2.0$ and $2.0-10 \mathrm{keV}$, the Chandra HETG light curve was extracted below $6 \AA$ and above $6 \AA$ and the NUSTAR one above and below $10 \mathrm{keV}$. Chandra hard and NUSTAR data show a strong periodic modulation, which can be seen in fig. 2. For visibility in fig. 2 the Chandra count rate was multiplied by a factor of 20 . To study this modulation quantitatively, we constructed Lomb-Scargle periodograms (LSPs) [12] of various data in soft and hard energy bands, which are presented in fig. 3. The top-left panel show the LSPs of hard (black) and soft (red) Swift XRT light curves. The highest peak of the hard LSP corresponds to the WD spin period - $351.33 \mathrm{~s}$. The spin modulation is not present in the LSP of the soft Swift XRT light curve: it shows peaks only at longer time scales with the strongest one at $5736 \mathrm{~s}$. Although QPOs in GK Per on a timescale of $\sim 5000$ were reported by many authors, this period is too close to the Swift orbital period of 5754 s to be distinguished from the windowing in the observations. The top-right and bottom-left panels of fig. 3 show the LSPs of the Chandra HETG and the NUSTAR light curves, respectively. There is no peak in the Chandra soft LSP at $5736 \mathrm{~s}$ 


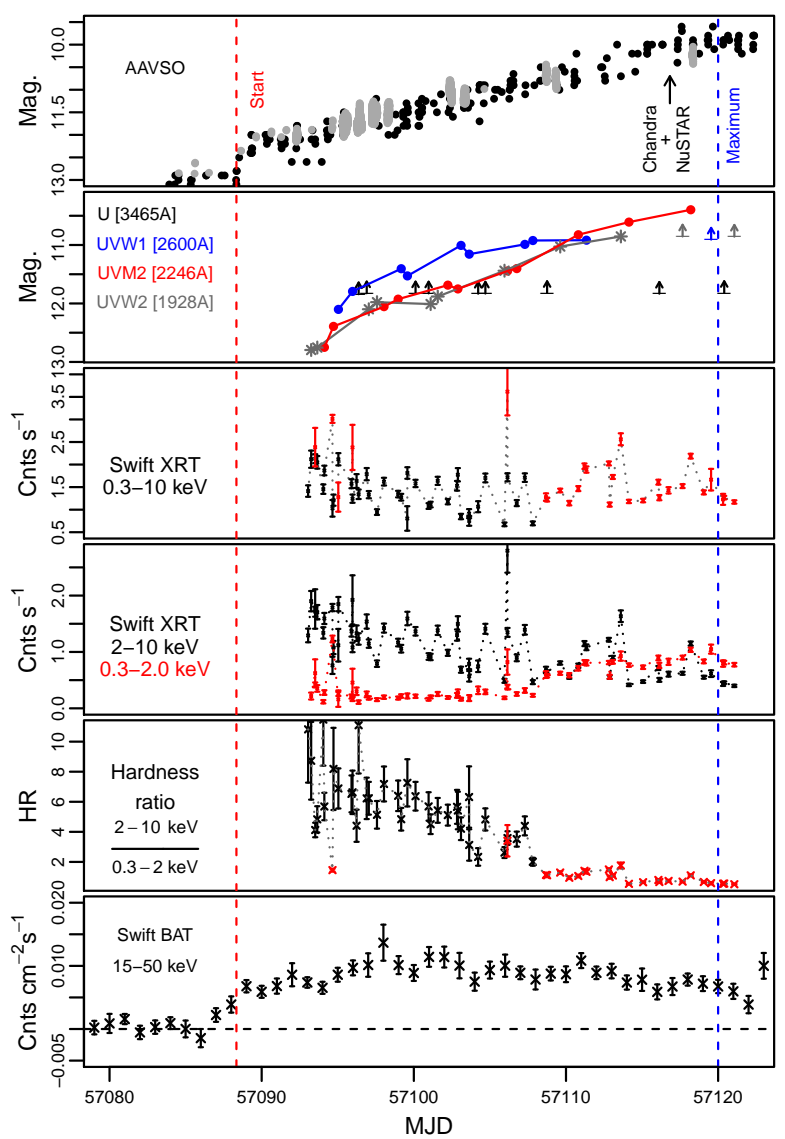

Figure 1: From top to bottom: AAVSO light curve in the $V$ band (grey) and without a filter (black). The red and blue vertical lines in all the panels mark the beginning (MJD 57088.34) and the maximum (MJD 570120) of the outburst in the optical band. The Swift UVOT light curves in different filters. The Swift XRT light curve in the PC (red) and WT (black) modes. The Swift XRT light curves above $2 \mathrm{keV}$ (black) and below $2 \mathrm{keV}$ (red). The X-ray hardness ratio from the data obtained in the WT (red) and PC (black) mode. The Swift BAT light curve.

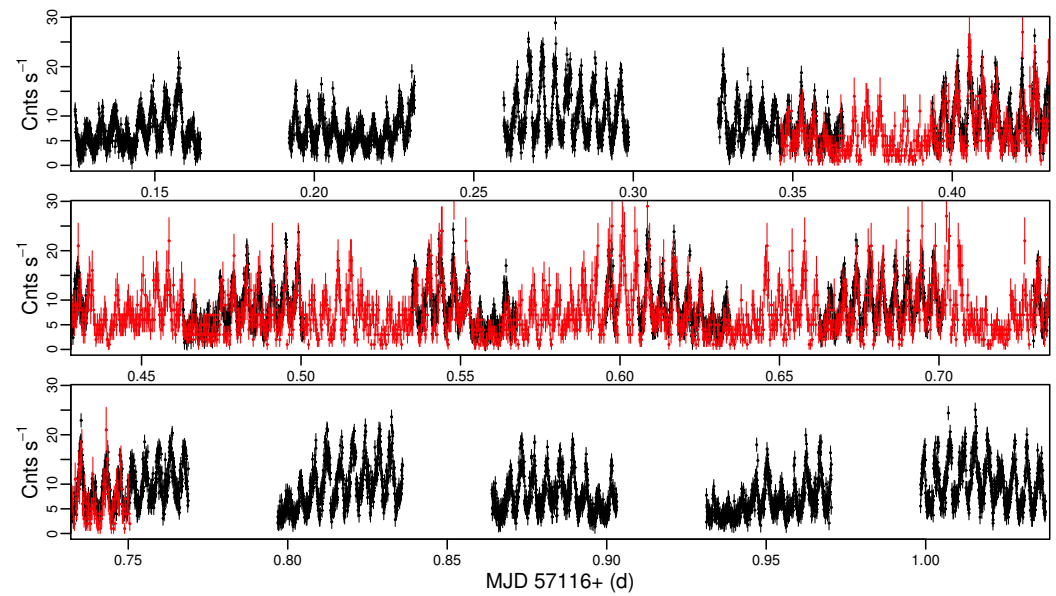

Figure 2: NUSTAR FPMA+FPMB light curve binned every $10 \mathrm{~s}$ (black) and the Chandra HETG light curve 1-6 Å wavelength range, binned every 20 s (red) and multiplied by a factor of 20 for visibility.. 
period, which confirms that this peak in the soft $S$ wift periodogram does not correspond to a real modulation. On the other hand, the NUSTAR and the hard Chandra LSPs show peaks at $\sim 7000$ s. These light curves are indeed variable on the timescales of kiloseconds with amplitudes up to 5 cnts $\mathrm{s}^{-1}$, as shown by fig. 2 .

All the LSPs of the light curves extracted above $2 \mathrm{keV}$ show a prominent peak corresponding to the WD spin period, while neither Chandra nor $S$ wift soft LSPs show any. The absence of the spin modulation in the region of $0.3-2 \mathrm{keV}$ may indicate that this emission component has a different origin and is visible during the whole spin cycle. The peak corresponding to the spin period is present even in the LSP of the NUSTAR light curve above $10 \mathrm{keV}$. Typically the spin modulation of IPs is not detected, or only marginally measurable, in the hard X-rays, since the cross section of the photoelectric absorption that usually causes the modulation decreases with energy. The effect of photoelectric absorption is not significant above $10 \mathrm{keV}$, so the observed high energy modulation might originate in a different mechanism than absorption of the accretion column emission. The comparison of the phase folded light NUSTAR light curves in two energy ranges (fig. 3) confirms that the spin modulation is not energy dependent: the spin profiles are almost identical.

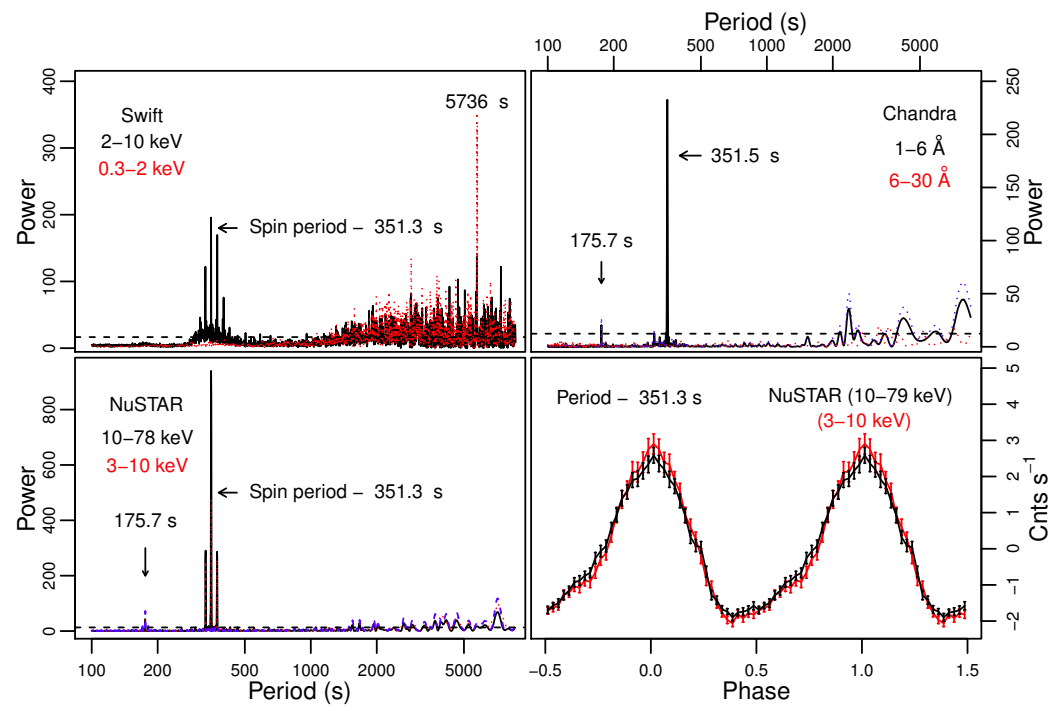

Figure 3: Top-left: the LSP of the $S$ wift XRT data in the $2-10 \mathrm{keV}$ energy range (black) and at $0.3-2 \mathrm{keV}$ (red). Top-right: the LSP of the Chandra HETG data in the energy range 1-6 $\AA$ before (red) and after (blue) subtracting the highest peak, at $351.5 \mathrm{~s}$. The red line shows the LSP of the Chandra HETG data in the energy range 6-30 A. Bottom-left: the LSP of the NuSTAR data in the $10-79 \mathrm{keV}$ (black) and $3-10 \mathrm{keV}$ (red) energy range. The blue line shows the LSP of the NuSTAR data in the $10-79 \mathrm{keV}$ range after subtracting the peak at $351.3 \mathrm{~s}$. The horizontal dashed lines show the $0.3 \%$ false alarm probability level at all the LSPs. Bottom-right: the NUSTAR light curve in the $3-10 \mathrm{keV}$ (red) and $10-79 \mathrm{keV}$ (black) ranges, folded with the WD spin period of $351.3 \mathrm{~s}$

\subsection{X-ray spectra}

From the timing analysis we found that there are at least two different sources of X-ray emission in GK Per: one dominates above $2 \mathrm{keV}$ and originates somewhere close to the $\mathrm{WD}$, since the flux in this range is modulated with the WD spin period, and the second source, dominating 
below $2 \mathrm{keV}$, is visible during the whole spin cycle. We first analysed the hard portion of the X-ray spectrum using the NUSTAR and Chandra HETG observations.

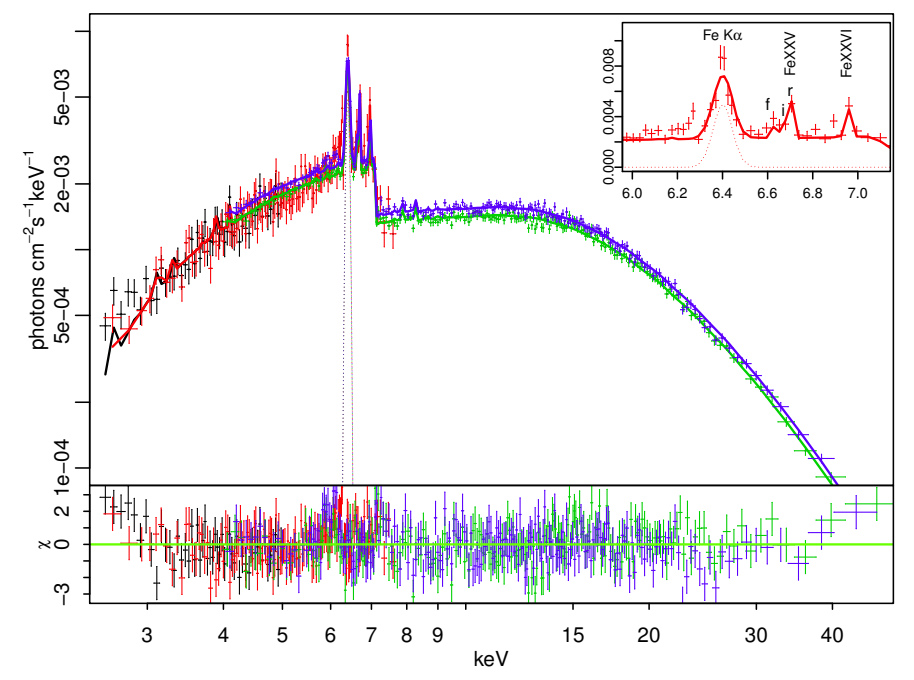

Figure 4: The NUSTAR FPMA (blue) and FPMB (green) and Chandra MEG (black) and HEG (red) spectra and the best-fitting model: constant $\times$ TBabs $\times$ pwab $\times$ (vmcflow + gaussian). The model components are marked with the dashed lines. The inset shows the Fe complex in the Chandra HEG spectrum.

We used the cooling flow model vmcf low, which calculates a plasma in collisional ionization equilibrium (CIE) with a range of temperatures and variable abundances of individual elements. The shape of continuum above $2 \mathrm{keV}$ indicates that the emission is highly absorbed. The best fit was obtained with the pwab model [13], in which the fraction of X-rays affected by a given column density $\mathrm{N}(\mathrm{H})$ is a power-law function of $\mathrm{N}(\mathrm{H})$ with index $\beta$. We also added a Gaussian component to fit the $\mathrm{Fe} \mathrm{K} \alpha$ fluorescent line at $6.4 \mathrm{keV}$. The model slightly underestimates the flux in the forbidden line of the Fe XXv triplet, which may indicate contribution of the photoinization processes. There are also residuals around 6.2-6.3 keV, suggesting Compton-downscattering of photons. The maximum plasma temperature was found to be $16.2 \mathrm{keV}$.

We then analyzed the spectrum below $2 \mathrm{keV}$ focusing on the Chandra HETG data (see fig. 5). From the Gaussian fits of all the the emission lines in the Chandra MEG spectrum we found that the $G$ ratio $(G=(f+i) / r$, where $r, i, f$ are the fluxes in the resonance, intercombination and forbidden line of the He-like triplets) is around 2, which means that there is no strong photoionizing component. In case of pure photoionized plasma $\mathrm{G}$ is $\sim 4$. We either have a collisional-ionization mechanism or a "hybrid plasma", a mixture of collisional and photionization [14]. The top panel of figure 5 shows the comparison of the Chandra MEG spectrum obtained in 2015 with Chandra MEG data discussed in [15]. The most recent spectrum of GK Per has much weaker lines in the region above $20 \AA$, which is due to contaminant build-up of the Chandra HETG+ACIS detector. The low energy effective area is reduced in 2015 compared to 2002. The Chandra spectra in fig. 5 give an additional proof that there are several distinct sources of emission: there is no correlation between the $6-11 \AA$ emission lines strengths and the hard continua below $5 \AA$. The complexity of the spectrum is demonstrated by the ratios of $\mathrm{H}$ to He-like lines, which in case of pure collisionalionization is a signature of the plasma temperature. The He-like lines are stronger than the H- 
like for all the species. The Si XIII to $\mathrm{Si}$ XIV lines ratio indicates a temperature $\sim 0.9 \mathrm{keV}$, so the origin of these lines is not in the hotter plasma that explains the NuSTAR spectrum. The He to $\mathrm{H}$-like lines ratios of $\mathrm{Mg}$ and $\mathrm{Ne}$ correspond to even lower plasma temperatures. These lines also cannot be explained by another mkcflow component at lower temperature since the cooling flow model always produces $\mathrm{H}$-like lines stronger than the He-like lines [16]. The middle and bottom panels of fig. 5 show the comparison of the Chandra MEG spectrum with the predictions of the single temperature thermal plasma emission model. We added to the best-fitting model of the NUSTAR data a vapec component (a single-temperature plasma in CIE with variable abundances of individual elements) and a Gaussian at $0.5 \mathrm{keV}$ to represent the $\mathrm{N}$ VII line. Following [18] and [19] we also introduced a blackbody component to represent the thermalized X-ray emission from the WD surface at $\mathrm{kT}=66 \mathrm{eV}$. In the middle panel of fig. 5 the temperature of the vapec component was fixed to $0.9 \mathrm{keV}$, in order fit the Si XIII to Si XIV lines ratio. In this case the model underestimates the level of continuum and overestimates the $\mathrm{He}$ to $\mathrm{H}$-like lines ratio of $\mathrm{Mg}$ and $\mathrm{Ne}$. In the bottom panel the temperature of the vapec component corresponds to the best-fitting value $-4.9 \mathrm{keV}$, which correctly estimates the level of continuum, but cannot reproduce the line ratios. A lower-temperature vapec component with higher normalization constant affected by a complex absorber could explain the Si lines and the continuum level, but not the He to H-like lines ratio of $\mathrm{Mg}$ and $\mathrm{Ne}$. The emission lines ratios clearly indicate a multi-temperature plasma emission. However, we added another apec component to fit the lines at longer wavelengths, but it did not improve the fit significantly. Thus, the overall spectrum below $2 \mathrm{keV}$ cannot be represented with a model of plasma in CIE, not with a cooling flow, neither with single or two-temperature vapec model.

\section{Discussion}

The NUSTAR observations of GK Per provided the first detection of a high amplitude modulation due to the WD spin period in X-rays above $10 \mathrm{keV}$ in an IP (only XY Ari in outbursts is known to show a comparable amplitude of modulation). The fact that the spin modulation is so strong in hard X-rays and that the pulse amplitude is not energy dependent indicate that the modulation is a geometric effect rather than due to absorption as in the majority of IPs. This modulation can be partially explained by an obscuration of the lower accretion pole by the inner disk [20,21]. However, the obscuration of the lower pole alone, does not explain the pulse profile. A small shock region with a low shock height will either be completely visible or completely behind the WD with very little transition in between, resulting in a square wave spin modulation. In case of GK Per the modulation is quasi-sinusoidal and about $40 \%$ of the total flux is always visible, suggesting a large shock height or an extended shock region. In the first case the soft X-rays, originating closer to the WD surface, will show more prominent modulation, while the hardest X-rays — just moderate eclipses. In GK Per the pulse profiles are not energy dependent, so we can reject this possibility. In GK Per we most probably deal with an accretion curtain whose footprint is very extended and forms an arc that covers $180 \mathrm{deg}$. The fraction of the arc that is visible can vary smoothly, resulting in only moderate energy dependence of pulses.

The spectrum above $2 \mathrm{keV}$ can be well fitted with the cooling flow model with maximum temperature of $16.2 \mathrm{keV}$, representing the emission from the WD accretion column. The continuum 


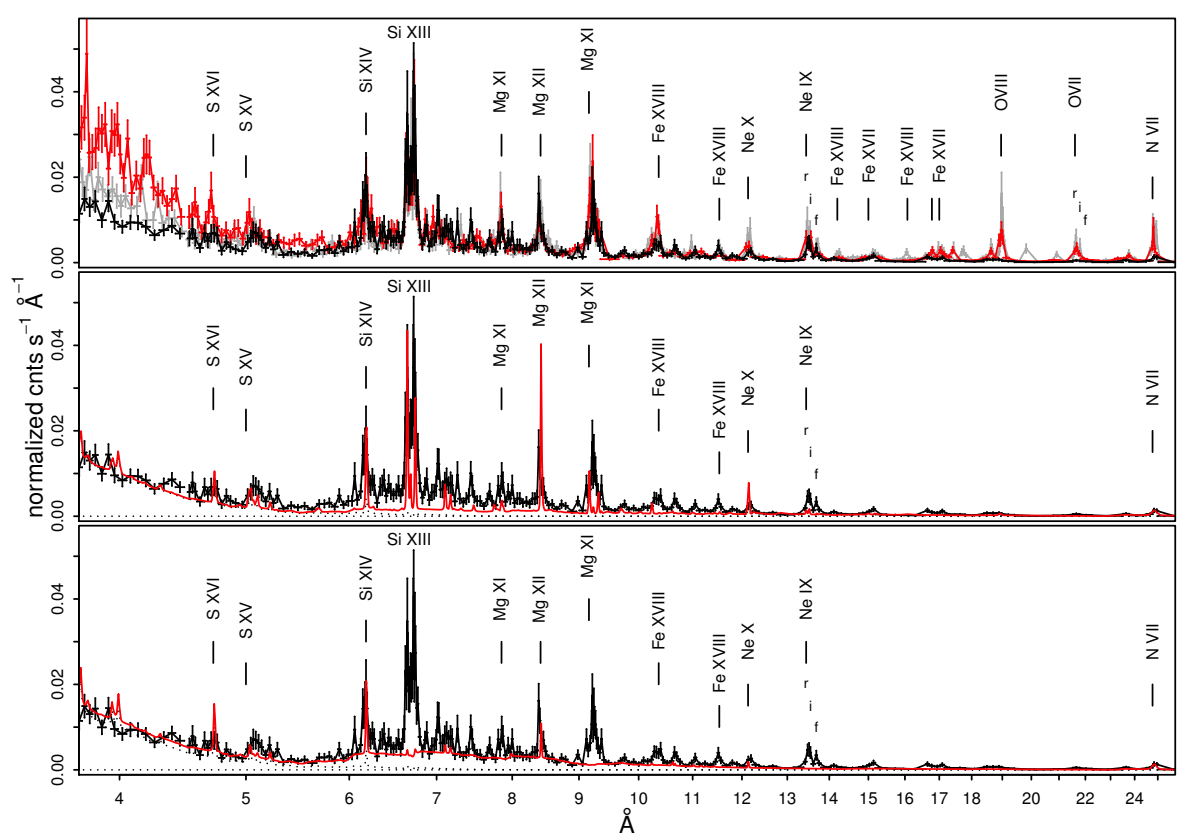

Figure 5: From top to bottom: comparison of the Chandra MEG spectra of GK Per in outbursts in 2015 and 2002. The Chandra MEG spectrum discussed in this paper is plotted in black, while the Chandra MEG spectra obtained on March 27 and April 92002 (PI C. Mauche) are plotted in red and grey, respectively. All the observations were performed close to the optical maxima. Middle panel: the 2015 Chandra MEG spectrum and the phabs $\times$ (pwab $\times$ (vmcflow + gaussian) + vapec + bb + gaussian) model (the red line). The temperature of the vapec component was fixed to $0.9 \mathrm{keV}$. Bottom panel: The 2015 Chandra MEG spectrum with the same model and the $4.9 \mathrm{keV}$ temperature of the vapec.

indicates that the source is highly absorbed, and this is also supported by the fact that the contribution of the cooling flow component to the observed line emission below $2 \mathrm{keV}$ should be negligible. The source of this absorption most probably is the pre-shock material. The shock temperature derived from the fit is lower than that observed in quiescence and at the beginning of the outburst, which is about $26-27 \mathrm{KeV}[22,23,24]$. When the inner radius of the accretion disk shrinks, the shock temperature is reduced, because the approximation of the free fall velocity cannot be used anymore [27]. The magnetospheric radius is defined by a balance between the ram pressure in the disk, which depends on mass transfer, and the magnetic pressure. As long as the optical flux is increasing, we expect the mass transfer to be constantly increasing as well, since the optical probes the portion of the disk involved in the outburst. This should result into gradual shrinking of the inner disk radius and lowering of the shock temperature.

We propose that the magnetospheric boundary is the emission site of the intermediate spectral component $(0.8-2 \mathrm{keV})$ and the intermediate energy $\mathrm{X}$-ray flux is related to the decrease of the shock temperature. If the shock temperature during outbursts is $2 / 3$ of that in quiescence [23, 25], half of the remaining energy is radiated away in the Keplerian disk. Where is the remaining 1/6th irradiated and how do we explain the energy budget? The site of emission may thus be the magnetospheric boundary, producing this intermediate X-ray spectral component, in the $0.8-2 \mathrm{keV}$ range, even if the exact mechanism of emission is not clear yet. The softest part of the spectrum can 
indeed be blackbody-like and originate on the surface of the WD, heated by the accretion column. Such blackbody-like component in an X-ray spectrum is a distinct property of "soft intermediate polars" (see e.g. [26]).

\section{Acknowledgements}

This work has been partially funded with an ASI-INAF I/037/12/0 award.

\section{References}

[1] Williams, A. S. 1901, Astronomische Nachrichten, 155, 29

[2] Sabbadin, F., \& Bianchini, A. 1983, A\&A, 54, 393

[3] Bianchini, A., Sabbadin, F., Favero, G. C., \& Dalmeri, I. 1986, A\&A, 160, 367

[4] Kim, S.-W., Wheeler, J. C., \& Mineshige, S. 1992, ApJ, 384, 269

[5] King, A. R., Ricketts, M. J., \& Warwick, R. S. 1979, MNRAS, 187, 77P

[6] Watson, M. G., King, A. R., \& Osborne, J. 1985, MNRAS, 212, 917

[7] Crampton, D., Fisher, W. A., \& Cowley, A. P. 1986, ApJ, 300, 788

[8] McLaughlin, D. B. 1960, in Stellar Atmospheres, ed. J. L. Greenstein, 585

[9] Warner, B. 1976, in IAU Symposium, Vol. 73, Structure and Evolution of Close Binary Systems, ed. P. Eggleton, S. Mitton, \& J. Whelan, 85

[10] Krimm, H. A., Holland, S. T., Corbet, R. H. D., et al. 2013, ApJS, 209, 33

[11] Šimon, V. 2015, A\&A, 575, A65

[12] Scargle, J. D., 1982, ApJ, 263, 835

[13] Done, C. \& Magdziarz, P., 1998, MNRAS, 298, 737

[14] Porquet, D. \& Dubau, J., 2000, AAPS, 143, 495

[15] Mukai, K., Kinkhabwala, A., Peterson, J. R., et al. 2003, ApJ, 586, L77

[16] Luna, G. J. M., Raymond, J. C., Brickhouse, N. S., et al., 2015, A\&A, 578, A15

[17] Hellier, C., Harmer, S., \& Beardmore, A. P. 2004, MNRAS, 349, 710

[18] Vrielmann, S., Ness, J.-U., \& Schmitt, J. H. M. M., 2005, A\&A, 439, 287

[19] Evans, P. A., Beardmore, A. P., Osborne, J. P., \& Wynn, G. A., 2009, MNRAS, 399, 1167

[20] Hellier, C., Mukai, K., Beardmore, A. P. 1997, MNRAS, 292, 397

[21] Hellier, C., Harmer, S., \& Beardmore, A. P. 2004, MNRAS, 349, 710

[22] Ishida, M., Sakao, T., Makishima, K., et al., 1992, MNRAS, 254, 647

[23] Brunschweiger, J., Greiner, J., Ajello, M., \& Osborne, J., 2009, A\&A, 496, 121

[24] Yuasa, T., Hayashi, T., \& Ishida, M. 2016, MNRAS, 459, 779

[25] Suleimanov, V., Doroshenko, V., Ducci, L., et al., 2016, A\&A, 591, A35

[26] Evans, P. A. \& Hellier, C., 2007, ApJ, 2007, 663, 1277 
[27] Suleimanov, V., Revnivtsev, M., \& Ritter, H., 2005, A\&A, 435, 191

\section{DISCUSSION}

VOITEK SIMON: Did you detect any changes of the profile and amplitude of the spin modulation with the progress of the outburst of GK Per?

POLINA ZEMKO: We tried to sum up every 5 observations, extract the light curve above 1.5 $\mathrm{keV}$, remove the trend and to fold the resultant light curves with the spin period. The amplitude of modulation was stable, but the spin profile became more smooth with time. 